\title{
Stochastic Comparison of Parallel Systems with Log-Lindley Distributed Components
}

\author{
Shovan Chowdhury \\ Indian Institute of Management, Kozhikode \\ Quantitative Methods and Operations Management Area \\ Kerala, India \\ Amarjit Kundu \\ Santipur College \\ Department of Mathematics \\ West Bengal, India
}

August 4, 2021

\begin{abstract}
In this paper, we study stochastic comparisons of parallel systems having log-Lindley distributed components. These comparisons are carried out with respect to reversed hazard rate and likelihood ratio ordering.
\end{abstract}

Keywords and Phrases: Likelihood ratio order, Log-Lindley distribution, Majorization, Multiple-outlier model, Reversed hazard rate order, Schur-convex.

AMS 2010 Subject Classifications: 62G30, 60E15, 60K10

\section{Introduction}

In reliability optimization and life testing experiments, many times the tests are censored or truncated when failure of a device during the warranty period may not be counted or items may be replaced after a certain time under a replacement policy. Moreover, many reliability systems and biological organism including human life span are bounded above because of test conditions, cost or other constraints. These situations result in a data set which is modeled by distributions with finite range (i.e. with bounded support) viz. power function density, finite range density, truncated Weibull, beta, Kumaraswamy and so on (see for example, Ghitany [5], Lai and Jones [10], Lai and Mukherjee [11], Moore and Lai [17] and Mukherjee and Islam [18]).

Recently, Gómez et al. [6] introduce the log-Lindley (LL) distribution with parameters $(\sigma, \lambda)$, written as $\operatorname{LL}(\sigma, \lambda)$, as an alternative to the beta distribution with the probability density 
function given by

$$
f(x ; \sigma, \lambda)=\frac{\sigma^{2}}{1+\lambda \sigma}(\lambda-\log x) x^{\sigma-1} ; 0<x<1, \lambda \geq 0, \sigma>0,
$$

where $\sigma$ is the shape parameter and $\lambda$ is the scale parameter. This distribution with a simple expression and nice reliability properties, is derived from the generalized Lindley distribution as proposed by Zakerzadeh and Dolati [21], which is again a generalization of the Lindley distribution as proposed by Lindley [14]. The LL distribution exhibits bath-tub failure rates and has increasing generalized failure rate (IGFR). This distribution has useful applications in the context of inventory management, pricing and supply chain contracting problems (see, for example, Ziya et al. [22], Lariviere and Porteus [12] and Lariviere [13]), where a demand distribution is required to have the IGFR property. Moreover, it has application in the actuarial context where the cumulative distribution function (CDF) of the LL distribution is used to distort the premium principle (Gómez et al. [6]). The LL distribution is also shown to fit rates and proportions data better than the beta distribution (Gómez et al. [6]).

Order statistics play an important role in reliability optimization, life testing, operations research and many other areas. Parallel and series systems are the building blocks of many complex coherent systems in reliability theory. While the lifetime of a series system corresponds to the smallest order statistic $X_{1: n}$, the same of a parallel system is represented by the largest order statistic $X_{n: n}$. Although stochastic comparisons of order statistics from homogeneous populations have been studied in detail in the literature, not much work is available so far for the same from heterogeneous populations, due to its complicated nature of expressions. Such comparisons are studied with exponential, gamma, Weibull, generalized exponential or Fréchet distributed components with unbounded support. One may refer to Dykstra et al. [2], Misra and Misra [16], Zhao and Balakrishnan ([23]), Torrado and Kochar [20], Kundu and Chowdhury [8], Kundu et al. [9], Gupta et al. [7] and the references there in. Moreover, not much attention has been paid so far to the stochastic comparison of two systems having finite range distributed components. The notion of majorization (Marshall et al. [5]) is also essential to the understanding of the stochastic inequalities for comparing order statistics. This concept is used in the context of optimal component allocation in parallel-series as well as in series-parallel systems, allocation of standby in series and parallel systems, and so on, see, for instance, ElNeweihi et al. [3]. It is also used in the context of minimal repair of two-component parallel system with exponentially distributed lifetime by Boland and El-Neweihi [1].

In this paper our main aim is to compare two parallel systems in terms of reversed hazard rate order and likelihood ratio order with majorized scale and shape parameters separately, when the components are from two heterogeneous LL distributions as well as from the multiple outlier LL random variables. The rest of the paper is organized as follows. In Section 2, we have given the required notations, definitions and some useful lemmas which have been used throughout the paper. Results related to reversed hazard rate ordering and likelihood ratio ordering between two order statistics $X_{n: n}$ and $Y_{n: n}$ are derived in Section 3. 
Throughout the paper, the word increasing (resp. decreasing) and nondecreasing (resp. nonincreasing) are used interchangeably, and $\Re$ denotes the set of real numbers $\{x:-\infty<x<$ $\infty\}$. We also write $a \stackrel{\text { sign }}{=} b$ to mean that $a$ and $b$ have the same sign. For any differentiable function $k(\cdot)$, we write $k^{\prime}(t)$ to denote the first derivative of $k(t)$ with respect to $t$.

\section{Notations, Definitions and Preliminaries}

For an absolutely continuous random variable $X$, we denote the probability density function, the distribution function and the reversed hazard rate function by $f_{X}(\cdot), F_{X}(\cdot)$, and $\tilde{r}_{X}(\cdot)$ respectively. The survival or reliability function of the random variable $X$ is written as $\bar{F}_{X}(\cdot)=1-F_{X}(\cdot)$.

In order to compare different order statistics, stochastic orders are used for fair and reasonable comparison. In literature many different kinds of stochastic orders have been developed and studied. The following well known definitions may be obtained in Shaked and Shanthiku$\operatorname{mar}[19]$.

Definition 2.1 Let $X$ and $Y$ be two absolutely continuous random variables with respective supports $\left(l_{X}, u_{X}\right)$ and $\left(l_{Y}, u_{Y}\right)$, where $u_{X}$ and $u_{Y}$ may be positive infinity, and $l_{X}$ and $l_{Y}$ may be negative infinity. Then, $X$ is said to be smaller than $Y$ in

(i) likelihood ratio (lr) order, denoted as $X \leq_{l r} Y$, if

$$
\frac{f_{Y}(t)}{f_{X}(t)} \text { is increasing int } \in\left(l_{X}, u_{X}\right) \cup\left(l_{Y}, u_{Y}\right) \text {; }
$$

(ii) hazard rate (hr) order, denoted as $X \leq_{h r} Y$, if

$$
\frac{\bar{F}_{Y}(t)}{\bar{F}_{X}(t)} \text { is increasing int } \in\left(-\infty, \max \left(u_{X}, u_{Y}\right)\right) \text {, }
$$

which can equivalently be written as $r_{X}(t) \geq r_{Y}(t)$ for all $t$;

(iii) reversed hazard rate (rhr) order, denoted as $X \leq_{r h r} Y$, if

$$
\frac{F_{Y}(t)}{F_{X}(t)} \text { is increasing int } \in\left(\min \left(l_{X}, l_{Y}\right), \infty\right) \text {, }
$$

which can equivalently be written as $\tilde{r}_{X}(t) \leq \tilde{r}_{Y}(t)$ for all $t$;

(iv) usual stochastic (st) order, denoted as $X \leq \leq_{\text {st }} Y$, if $\bar{F}_{X}(t) \leq \bar{F}_{Y}(t)$ for all $t \in(-\infty, \infty)$.

In the following diagram we present a chain of implications of the stochastic orders, see, for instance, Shaked and Shanthikumar [19], where the definitions and usefulness of these orders can be found. 


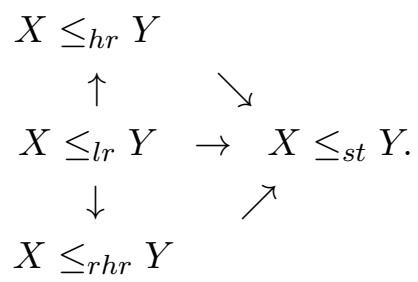

It is well known that the results on different stochastic orders can be established on using majorization order(s). Let $I^{n}$ denotes an $n$-dimensional Euclidean space where $I \subseteq \Re$. Further, let $\mathbf{x}=\left(x_{1}, x_{2}, \ldots, x_{n}\right) \in I^{n}$ and $\mathbf{y}=\left(y_{1}, y_{2}, \ldots, y_{n}\right) \in I^{n}$ be any two real vectors with $x_{(1)} \leq x_{(2)} \leq \cdots \leq x_{(n)}$ being the increasing arrangements of the components of the vector $\mathbf{x}$. The following definitions may be found in Marshall et al. [15].

Definition 2.2 The vector $\mathbf{x}$ is said to majorize the vector $\mathbf{y}$ (written as $\mathbf{x} \succeq \stackrel{m}{\succeq} \mathbf{y}$ ) if

$$
\sum_{i=1}^{j} x_{(i)} \leq \sum_{i=1}^{j} y_{(i)}, j=1,2, \ldots, n-1, \text { and } \sum_{i=1}^{n} x_{(i)}=\sum_{i=1}^{n} y_{(i)} .
$$

Definition 2.3 A function $\psi: I^{n} \rightarrow \Re$ is said to be Schur-convex (resp. Schur-concave) on $I^{n}$ if

$$
\mathbf{x} \stackrel{m}{\succeq} \mathbf{y} \text { implies } \psi(\mathbf{x}) \geq(\text { resp. } \leq) \psi(\mathbf{y}) \text { for all } \mathbf{x}, \mathbf{y} \in I^{n}
$$

Notation 2.1 Let us introduce the following notations.

(i) $\mathcal{D}_{+}=\left\{\left(x_{1}, x_{2}, \ldots, x_{n}\right): x_{1} \geq x_{2} \geq \ldots \geq x_{n}>0\right\}$.

(ii) $\mathcal{E}_{+}=\left\{\left(x_{1}, x_{2}, \ldots, x_{n}\right): 0<x_{1} \leq x_{2} \leq \ldots \leq x_{n}\right\}$.

Next, two lemmas are given which will be used to prove our main results. The first one can be obtained by combining Proposition H2 of Marshall et al. ([15], p. 132) and Lemma 3.2 of Kundu et al. ([9]) while the second one is due to Lemma 3.4 of Kundu et al. ([9]).

Lemma 2.1 Let $\varphi(\mathbf{x})=\sum_{i=1}^{n} g_{i}\left(x_{i}\right)$ with $\mathbf{x} \in \mathcal{D}_{+}$, where $g_{i}: \mathbb{R} \rightarrow \mathbb{R}$ is differentiable, for all $i=1,2, \ldots, n$. Then $\varphi(\mathbf{x})$ is Schur-convex (Schur-concave) on $\mathcal{D}_{+}$if, and only if,

$$
g_{i}^{\prime}(a) \geq(\text { resp. } \leq) g_{i+1}^{\prime}(b) \text { whenever } a \geq b, \text { for all } i=1,2, \ldots, n-1,
$$

where $g^{\prime}(a)=\left.\frac{d g(x)}{d x}\right|_{x=a}$.

Lemma 2.2 Let $\varphi(\mathbf{x})=\sum_{i=1}^{n} g_{i}\left(x_{i}\right)$ with $\mathbf{x} \in \mathcal{E}_{+}$, where $g_{i}: \mathbb{R} \rightarrow \mathbb{R}$ is differentiable, for all $i=1,2, \ldots, n$. Then $\varphi(\mathbf{x})$ is Schur-convex (Schur-concave) on $\mathcal{E}_{+}$if, and only if,

$$
g_{i+1}^{\prime}(a) \geq(\text { resp. } \leq) g_{i}^{\prime}(b) \text { whenever } a \geq b, \text { for all } i=1,2, \ldots, n-1,
$$

where $g^{\prime}(a)=\left.\frac{d g(x)}{d x}\right|_{x=a}$. 


\section{Main Results}

For $i=1,2, \ldots, n$, let $X_{i}$ (resp. $Y_{i}$ ) be $n$ independent nonnegative random variables following LL distribution as given in (1.1).

If $F_{n: n}(\cdot)$ and $G_{n: n}(\cdot)$ be the distribution functions of $X_{n: n}$ and $Y_{n: n}$ respectively, where $\boldsymbol{\sigma}=\left(\sigma_{1}, \sigma_{2}, \ldots, \sigma_{n}\right), \boldsymbol{\theta}=\left(\theta_{1}, \theta_{2}, \ldots, \theta_{n}\right), \boldsymbol{\lambda}=\left(\lambda_{1}, \lambda_{2}, \ldots, \lambda_{n}\right)$ and $\boldsymbol{\delta}=\left(\delta_{1}, \delta_{2}, \ldots, \delta_{n}\right)$, then

$$
F_{n: n}(x)=\prod_{i=1}^{n} \frac{x^{\sigma_{i}}\left(1+\sigma_{i}\left(\lambda_{i}-\log x\right)\right)}{1+\lambda_{i} \sigma_{i}}
$$

and

$$
G_{n: n}(x)=\prod_{i=1}^{n} \frac{x^{\theta_{i}}\left(1+\theta_{i}\left(\delta_{i}-\log x\right)\right)}{1+\delta_{i} \theta_{i}} .
$$

Again, if $\tilde{r}_{n: n}^{X}$ and $\tilde{r}_{n: n}^{Y}$ are the reversed hazard rate functions of $X_{n: n}$ and $Y_{n: n}$ respectively, then

$$
\tilde{r}_{n: n}^{X}(x)=\sum_{i=1}^{n} \frac{\sigma_{i}}{x}\left(1-\frac{1}{1+\sigma_{i}\left(\lambda_{i}-\log x\right)}\right)
$$

and

$$
\tilde{r}_{n: n}^{Y}(x)=\sum_{i=1}^{n} \frac{\theta_{i}}{x}\left(1-\frac{1}{1+\theta_{i}\left(\delta_{i}-\log x\right)}\right) .
$$

The following two theorems show that under certain conditions on parameters, there exists reversed hazard rate ordering between $X_{n: n}$ and $Y_{n: n}$.

Theorem 3.1 For $i=1,2, \ldots, n$, let $X_{i}$ and $Y_{i}$ be two sets of mutually independent random variables with $X_{i} \sim L L\left(\sigma_{i}, \lambda_{i}\right)$ and $Y_{i} \sim L L\left(\theta_{i}, \lambda_{i}\right)$. Further, suppose that $\boldsymbol{\sigma}, \boldsymbol{\theta}, \boldsymbol{\lambda} \in \mathcal{D}_{+}$or $\boldsymbol{\sigma}, \boldsymbol{\theta}, \boldsymbol{\lambda} \in \mathcal{E}_{+}$. Then,

$$
\boldsymbol{\sigma} \succeq \boldsymbol{m} \text { implies } X_{n: n} \geq_{r h r} Y_{n: n}
$$

Proof: Let $g_{i}(y)=\frac{y}{x}\left(1-\frac{1}{1+y\left(\lambda_{i}-\log x\right)}\right)$. Differentiating $g_{i}(y)$ with respect to $y$, we get

$$
g_{i}^{\prime}(y)=\frac{1}{x}\left(1-\frac{1}{\left(1+y\left(\lambda_{i}-\log x\right)\right)^{2}}\right),
$$

giving

$$
g_{i}^{\prime}\left(\sigma_{i}\right)-g_{i+1}^{\prime}\left(\sigma_{i+1}\right)=\frac{\left(1+\sigma_{i}\left(\lambda_{i}-\log x\right)\right)^{2}-\left(1+\sigma_{i+1}\left(\lambda_{i+1}-\log x\right)\right)^{2}}{x\left(\left(1+\sigma_{i}\left(\lambda_{i}-\log x\right)\right)\left(1+\sigma_{i+1}\left(\lambda_{i+1}-\log x\right)\right)\right)^{2}} .
$$

So, if $\boldsymbol{\sigma}, \boldsymbol{\lambda} \in \mathcal{D}_{+}\left(\right.$resp. $\left.\mathcal{E}_{+}\right)$, then $g_{i}^{\prime}\left(\sigma_{i}\right)-g_{i+1}^{\prime}\left(\sigma_{i+1}\right) \geq(\leq) 0$. Then, by Lemma 2.1 (Lemma 2.2), $\tilde{r}_{n: n}^{X}(x)$ is Schur convex in $\boldsymbol{\sigma}$, proving the result.

The counterexample given below shows that the ascending (descending) order of the components of the scale and shape parameters are necessary for the result of Theorem 3.1 to hold. 


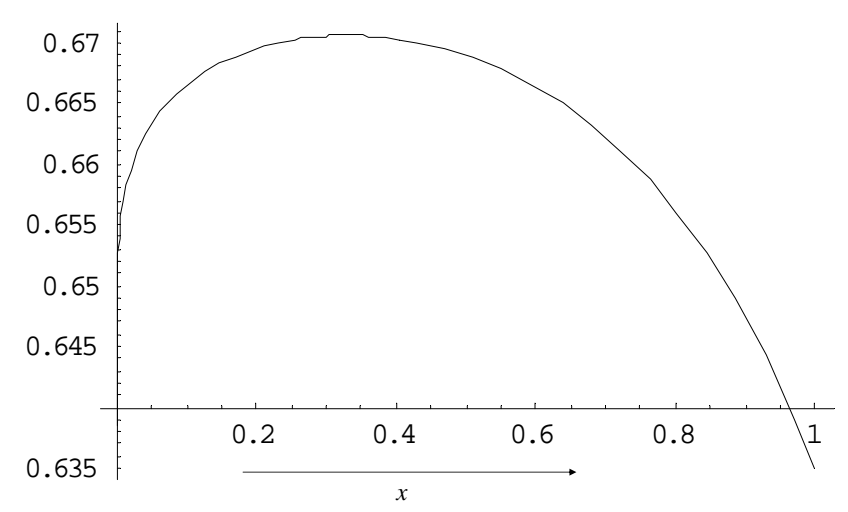

Figure 1: Graph of $\frac{F_{3: 3}(x)}{G_{3: 3}(x)}$

Counterexample 3.1 Let $X_{i} \sim L L\left(\sigma_{i}, \lambda_{i}\right)$ and $Y_{i} \sim L L\left(\theta_{i}, \lambda_{i}\right), i=1,2,3$. Now, if $\left(\sigma_{1}, \sigma_{2}, \sigma_{3}\right)=$ $(1,1,5) \in \mathcal{E}_{+},\left(\theta_{1}, \theta_{2}, \theta_{3}\right)=(1,2,4) \in \mathcal{E}_{+}$and $\left(\lambda_{1}, \lambda_{2}, \lambda_{3}\right)=(4,3,0.2) \in \mathcal{D}_{+}$are taken, then from Figure 1, it is clear that $\frac{F_{3: 3}(x)}{G_{3: 3}(x)}$ is not monotone, giving that $X_{3: 3} \Varangle_{r h r} Y_{3: 3}$, although $\boldsymbol{\sigma} \succeq \boldsymbol{m}$

Theorem 3.1 guarantees that for parallel systems of components having independent LL distributed lifetimes with common scale parameter vector, the majorized shape parameter vector leads to larger system's life in the sense of the reversed hazard rate ordering. Now the question arises-what will happen if the scale parameter $\boldsymbol{\lambda}$ majorizes $\boldsymbol{\delta}$ when the shape parameter vector remains constant? The theorem given below answers that if the order of the components of shape and scale parameter vectors are reversed, then $X_{n: n}$ will be smaller than $Y_{n: n}$ in reversed hazard rate ordering.

Theorem 3.2 For $i=1,2, \ldots, n$, let $X_{i}$ and $Y_{i}$ be two sets of mutually independent random variables with $X_{i} \sim L L\left(\sigma_{i}, \lambda_{i}\right)$ and $Y_{i} \sim L L\left(\sigma_{i}, \delta_{i}\right)$. Further, suppose that $\boldsymbol{\sigma} \in \mathcal{E}_{+}, \boldsymbol{\lambda}, \boldsymbol{\delta} \in \mathcal{D}_{+}$ or $\boldsymbol{\sigma} \in \mathcal{D}_{+}, \boldsymbol{\lambda}, \boldsymbol{\delta} \in \mathcal{E}_{+}$. Then,

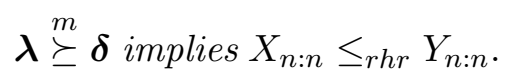

Proof: For $i=1,2 \ldots, n$, let us consider $g_{i}(y)=\frac{\sigma_{i}}{x}\left(1-\frac{1}{1+\sigma_{i}(y-\log x)}\right)$. Differentiating $g_{i}(y)$ with respect to $y$, we get

$$
g_{i}^{\prime}(y)=\frac{\sigma_{i}^{2}}{x\left(1+\sigma_{i}(y-\log x)\right)^{2}},
$$

giving

$$
\begin{aligned}
g_{i}^{\prime}\left(\lambda_{i}\right)-g_{i+1}^{\prime}\left(\lambda_{i+1}\right) \stackrel{\text { sign }}{=} & \left(\sigma_{i}^{2}-\sigma_{i+1}^{2}\right)+\sigma_{i}^{2} \sigma_{i+1}^{2}\left[\left(\lambda_{i+1}-\log x\right)^{2}-\left(\lambda_{i}-\log x\right)^{2}\right] \\
+ & 2 \sigma_{i} \sigma_{i+1}\left[\left(\sigma_{i} \lambda_{i+1}-\sigma_{i+1} \lambda_{i}\right)-\log x\left(\sigma_{i}-\sigma_{i+1}\right)\right] .
\end{aligned}
$$


So, if $\boldsymbol{\lambda} \in \mathcal{D}_{+}\left(\right.$resp. $\left.\mathcal{E}_{+}\right)$and $\boldsymbol{\sigma} \in \mathcal{E}_{+}\left(\right.$resp. $\left.\mathcal{D}_{+}\right)$, then $g_{i}^{\prime}\left(\lambda_{i}\right)-g_{i+1}^{\prime}\left(\lambda_{i+1}\right) \leq(\geq) 0$. So, by Lemma 2.1 (Lemma 2.2), $\tilde{r}_{n: n}^{X}(x)$ is Schur-concave in $\boldsymbol{\lambda}$, proving the result.

Next, one counterexample is provided to show that, nothing can be said about reversed hazard rate ordering between $X_{n: n}$ and $Y_{n: n}$ if $\boldsymbol{\lambda}$ majorizes $\boldsymbol{\delta}$ and all of $\boldsymbol{\lambda}, \boldsymbol{\delta}$ and $\boldsymbol{\sigma}$ are either in $\mathcal{E}_{+}$or in $\mathcal{D}_{+}$.

Counterexample 3.2 Let $X_{i} \sim L L\left(\sigma_{i}, \lambda_{i}\right)$ and $Y_{i} \sim\left(\sigma_{i}, \delta_{i}\right), i=1,2,3$. Let $\left(\lambda_{1}, \lambda_{2}, \lambda_{3}\right)=$ $(0.1,0.3,4.1) \in \mathcal{E}_{+}$and $\left(\delta_{1}, \delta_{2}, \delta_{3}\right)=(0.2,0.3,4) \in \mathcal{E}_{+}$, giving $\boldsymbol{\lambda} \stackrel{m}{\succeq} \boldsymbol{\delta}$. Now, if $\left(\sigma_{1}, \sigma_{2}, \sigma_{3}\right)=$ $(0.1,3,5) \in \mathcal{E}_{+}$is taken, then Figure 2 (a) shows that $\frac{F_{3: 3}(x)}{G_{3: 3}(x)}$ is increasing in $x$. Again if $\left(\sigma_{1}, \sigma_{2}, \sigma_{3}\right)=(2,3,5) \in \mathcal{E}_{+}$is taken, then Figure $2(b)$ shows that $\frac{F_{3: 3}(x)}{G_{3: 3}(x)}$ is decreasing in $x$. So, it can be concluded that, for all $\boldsymbol{\sigma}, \boldsymbol{\lambda}, \boldsymbol{\delta} \in \mathcal{D}_{+}\left(\right.$resp. $\left.\mathcal{E}_{+}\right), \boldsymbol{\lambda} \succeq \stackrel{m}{\succeq}$ does not always imply $X_{3: 3} \leq_{r h r} Y_{3: 3}$.

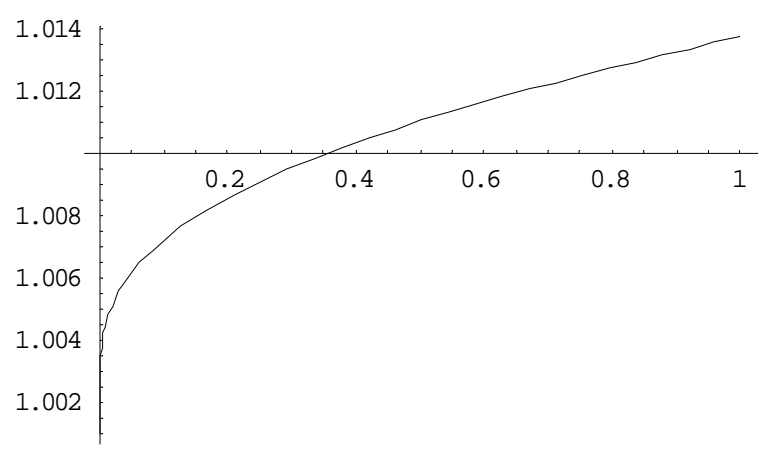

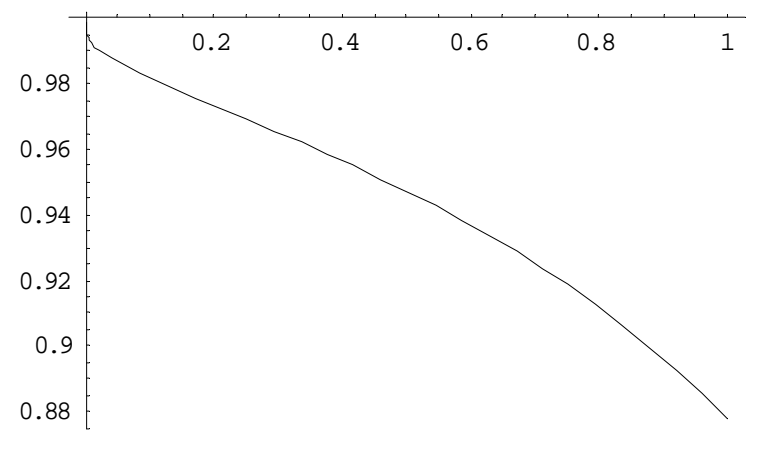

(b) For $\left(\sigma_{1}, \sigma_{2}, \sigma_{3}\right)=(2,3,5)$

(a) For $\left(\sigma_{1}, \sigma_{2}, \sigma_{3}\right)=(0.1,3,5)$

Figure 2: Graph of $\frac{F_{3: 3}(x)}{G_{3: 3}(x)}$

The following theorem shows that depending upon certain conditions, majorization order of the shape parameters implies likelihood ratio ordering between $X_{n: n}$ and $Y_{n: n}$.

Theorem 3.3 For $i=1,2, \ldots, n$, let $X_{i}$ and $Y_{i}$ be two sets of mutually independent random variables with $X_{i} \sim L L\left(\sigma_{i}, \lambda_{i}\right)$ and $Y_{i} \sim L L\left(\theta_{i}, \lambda_{i}\right)$. Further, suppose that $\boldsymbol{\sigma}, \boldsymbol{\theta}, \boldsymbol{\lambda} \in \mathcal{D}_{+}$or $\boldsymbol{\sigma}, \boldsymbol{\theta}, \boldsymbol{\lambda} \in \mathcal{E}_{+}$. Then, if $\lambda_{i} \sigma_{i}>1 / 2$,

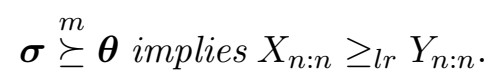

\section{Proof:}


In view of theorem 3.1 and using (3.1) and (3.2), here we have only to show that

$$
\begin{aligned}
\frac{\tilde{r}_{n: n}^{X}(x)}{\tilde{r}_{n: n}^{Y}(x)} & =\frac{\sum_{k=1}^{n} u_{k}\left(\sigma_{k}, x\right)}{\sum_{k=1}^{n} u_{k}\left(\theta_{k}, x\right)} \\
& =\eta(x)(\text { say }),
\end{aligned}
$$

is increasing in $x$, where $u_{k}(y, x)=\frac{y^{2}\left(\lambda_{k}-\log x\right)}{1+y\left(\lambda_{k}-\log x\right)}$. Now, differentiating $\eta(x)$ with respect to $x$,

$$
\begin{gathered}
\eta^{\prime}(x) \stackrel{\text { sign }}{=} \sum_{k=1}^{n} \frac{\partial u_{k}\left(\sigma_{k}, x\right)}{\partial x} \sum_{k=1}^{n} u_{k}\left(\theta_{k}, x\right)-\sum_{k=1}^{n} \frac{\partial u_{k}\left(\theta_{k}, x\right)}{\partial x} \sum_{k=1}^{n} u_{k}\left(\sigma_{k}, x\right) \\
=-h(\boldsymbol{\sigma}, x) \sum_{k=1}^{n} u_{k}\left(\theta_{k}, x\right)+h(\boldsymbol{\theta}, x) \sum_{k=1}^{n} u_{k}\left(\sigma_{k}, x\right),
\end{gathered}
$$

where

$$
h(\boldsymbol{\sigma}, x)=-\sum_{k=1}^{n} \frac{\partial u_{k}\left(\sigma_{k}, x\right)}{\partial x}=\frac{1}{x} \sum_{k=1}^{n} \frac{\sigma_{k}^{2}}{\left(1+\sigma_{k}\left(\lambda_{k}-\log x\right)\right)^{2}}
$$

and

$$
h(\boldsymbol{\theta}, x)=-\sum_{k=1}^{n} \frac{\partial u_{k}\left(\theta_{k}, x\right)}{\partial x}=\frac{1}{x} \sum_{k=1}^{n} \frac{\theta_{k}^{2}}{\left(1+\theta_{k}\left(\lambda_{k}-\log x\right)\right)^{2}} .
$$

Thus, to show that $\eta(x)$ is increasing in $x$, we have only to show that

$$
\psi(\boldsymbol{\sigma}, x)=\frac{h(\boldsymbol{\sigma}, x)}{\sum_{k=1}^{n} u_{k}\left(\sigma_{k}, x\right)}
$$

is Schur-concave in $\boldsymbol{\sigma}$.

Now, as

$$
\frac{\partial h(\boldsymbol{\sigma}, x)}{\partial \sigma_{i}}=\frac{1}{x} \cdot \frac{2 \sigma_{i}}{\left(1+\sigma_{i}\left(\lambda_{i}-\log x\right)\right)^{3}}
$$

and

$$
\frac{\partial}{\partial \sigma_{i}}\left[\sum_{k=1}^{n} u_{k}\left(\sigma_{k}, x\right)\right]=1-\frac{1}{\left(1+\sigma_{i}\left(\lambda_{i}-\log x\right)\right)^{2}},
$$

then

$$
\frac{\partial \psi}{\partial \sigma_{i}} \stackrel{\text { sign }}{=} \frac{2 \sigma_{i}}{\left(1+\sigma_{i}\left(\lambda_{i}-\log x\right)\right)^{3}} \sum_{k=1}^{n} u_{k}\left(\sigma_{k}, x\right)-x \cdot h(\boldsymbol{\sigma}, x)\left(1-\frac{1}{\left(1+\sigma_{i}\left(\lambda_{i}-\log x\right)\right)^{2}}\right) .
$$

So, if $\boldsymbol{\sigma}, \boldsymbol{\lambda} \in \mathcal{D}_{+}\left(\right.$resp $\left.\in \mathcal{E}_{+}\right)$, i.e., for $i \leq j$ if $\sigma_{i} \geq \sigma_{j}$ and $\lambda_{i} \geq \lambda_{j}\left(\sigma_{i} \leq \sigma_{j}, \lambda_{i} \leq \lambda_{j}\right)$, then noticing the fact that $\frac{1}{\left(1+\sigma_{i}\left(\lambda_{i}-\log x\right)\right)^{2}}$ is decreasing in $\sigma_{i}$ as well as in $\lambda_{i}$, it can be written that

$$
\frac{1}{\left(1+\sigma_{i}\left(\lambda_{i}-\log x\right)\right)^{2}} \leq(\geq) \frac{1}{\left(1+\sigma_{j}\left(\lambda_{i}-\log x\right)\right)^{2}} \leq(\geq) \frac{1}{\left(1+\sigma_{j}\left(\lambda_{j}-\log x\right)\right)^{2}}
$$

Again, as $\sigma_{i} \lambda_{i}>\frac{1}{2}$ implying $\sigma_{i}\left(\lambda_{i}-\log x\right)>\frac{1}{2}$ for all $0<x<1$, then

$$
\frac{\partial}{\partial \sigma_{i}}\left(\frac{\sigma_{i}}{\left(1+\sigma_{i}\left(\lambda_{i}-\log x\right)\right)^{3}}\right)=\frac{1-2 \sigma_{i}\left(\lambda_{i}-\log x\right)}{\left(1+\sigma_{i}\left(\lambda_{i}-\log x\right)\right)^{4}}<0,
$$


proving that $\frac{\sigma_{i}}{\left(1+\sigma_{i}\left(\lambda_{i}-\log x\right)^{3}\right.}$ is decreasing in $\sigma_{i}$. Again, it is also decreasing in $\lambda_{i}$. Thus, for all $\sigma_{i} \geq \sigma_{j}$ and $\lambda_{i} \geq \lambda_{j}\left(\sigma_{i} \leq \sigma_{j}, \lambda_{i} \leq \lambda_{j}\right)$,

$$
\frac{\sigma_{i}}{\left(1+\sigma_{i}\left(\lambda_{i}-\log x\right)\right)^{3}} \leq(\geq) \frac{\sigma_{j}}{\left(1+\sigma_{j}\left(\lambda_{i}-\log x\right)\right)^{3}} \leq(\geq) \frac{\sigma_{j}}{\left(1+\sigma_{j}\left(\lambda_{j}-\log x\right)\right)^{3}} .
$$

So, for all $i \leq j$

$$
\begin{aligned}
\frac{\partial \psi}{\partial \sigma_{i}}-\frac{\partial \psi}{\partial \sigma_{j}} \stackrel{\text { sign }}{=} \sum_{k=1}^{n} \frac{\sigma_{k}^{2}\left(\lambda_{k}-\log x\right)}{1+\sigma_{k}\left(\lambda_{k}-\log x\right)}\left[\frac{2 \sigma_{i}}{\left(1+\sigma_{i}\left(\lambda_{i}-\log x\right)\right)^{3}}-\frac{2 \sigma_{j}}{\left(1+\sigma_{j}\left(\lambda_{j}-\log x\right)\right)^{3}}\right] \\
+\sum_{k=1}^{n} \frac{\sigma_{k}^{2}}{\left(1+\sigma_{k}\left(\lambda_{k}-\log x\right)\right)^{2}}\left[\frac{1}{\left(1+\sigma_{i}\left(\lambda_{i}-\log x\right)\right)^{2}}-\frac{1}{\left(1+\sigma_{j}\left(\lambda_{j}-\log x\right)\right)^{2}}\right] \\
\leq(\geq) 0 .
\end{aligned}
$$

Thus the result follows from Lemma 3.1 (Lemma 3.3) of Kundu et al. ([9]).

Although Theorem 3.3 holds under a sufficient condition for two $n$ component systems, the next theorem shows that no such condition is required for these systems having multiple-outlier LL model if the scale parameter vectors of these systems are common.

Theorem 3.4 For $i=1,2, \ldots, n$, let $X_{i}$ and $Y_{i}$ be two sets of independent random variables each following the multiple-outlier $E W$ model such that $X_{i} \sim L L(\sigma, \lambda)$ and $Y_{i} \sim L L(\theta, \lambda)$ for $i=1,2, \ldots, n_{1}, X_{i} \sim L L\left(\sigma^{*}, \lambda^{*}\right)$ and $Y_{i} \sim L L\left(\theta^{*}, \lambda^{*}\right)$ for $i=n_{1}+1, n_{1}+2, \ldots, n_{1}+n_{2}(=n)$ If

$$
(\underbrace{\sigma, \sigma, \ldots, \sigma}_{n_{1}}, \underbrace{\sigma^{*}, \sigma^{*}, \ldots, \sigma^{*}}_{n_{2}}) \succeq(\underbrace{\theta, \theta, \ldots, \theta}_{n_{1}} \underbrace{\theta^{*}, \theta^{*}, \ldots, \theta^{*}}_{n_{2}})
$$

and either $\left\{\sigma \geq \sigma^{*}, \theta \geq \theta^{*}, \lambda \geq \lambda^{*}\right\}$ or $\left\{\sigma \leq \sigma^{*}, \theta \leq \theta^{*}, \lambda \leq \lambda^{*}\right\}$ then $X_{n: n} \geq_{l r} Y_{n: n}$.

Proof: Following Theorem 3.3 and in view of Theorem 3.1, we have only to show that

$$
\psi_{1}(\boldsymbol{\sigma}, x)=\frac{\sum_{k=1}^{n} \frac{\sigma_{k}^{2}}{\left(1+\sigma_{k}\left(\lambda_{k}-\log x\right)\right)^{2}}}{\sum_{k=1}^{n} \frac{\sigma_{k}^{2}\left(\lambda_{k}-\log x\right)}{1+\sigma_{k}\left(\lambda_{k}-\log x\right)}}
$$

is Schur-concave in $\boldsymbol{\sigma}$.

Now, three cases may arise:

Case( $i$ ) If $1 \leq i<j \leq n_{1}$, i.e., if $\sigma_{i}=\sigma_{j}=\sigma$ and $\lambda_{i}=\lambda_{j}=\lambda$, then $\frac{\partial \psi_{1}}{\partial \sigma_{i}}-\frac{\partial \psi_{1}}{\partial \sigma_{j}}=0$.

Case(ii) If $n_{1}+1 \leq i<j \leq n$, i.e., if $\sigma_{i}=\sigma_{j}=\sigma^{*}$ and $\lambda_{i}=\lambda_{j}=\lambda^{*}$, then $\frac{\partial \Psi}{\partial \sigma_{i}}-\frac{\partial \Psi}{\partial \sigma_{j}}=0$.

Case(iii) If $1 \leq i \leq n_{1}$ and $n_{1}+1 \leq j \leq n$, then $\sigma_{i}=\sigma, \lambda_{i}=\lambda$ and $\sigma_{j}=\sigma^{*}, \lambda_{i}=\lambda^{*}$. It can be easily shown that

$$
\begin{aligned}
\frac{\partial \psi_{1}}{\partial \sigma_{i}}-\frac{\partial \psi_{1}}{\partial \sigma_{j}} \stackrel{\text { sign }}{=} & \left(\frac{n_{1} \sigma^{2}}{\left(1+\xi_{1}\right)^{2}}+\frac{n_{2} \sigma^{* 2}}{\left(1+\xi_{2}\right)^{2}}\right)\left(\frac{\xi_{2}^{2}}{\left(1+\xi_{2}\right)^{2}}-\frac{\xi_{1}^{2}}{\left(1+\xi_{1}\right)^{2}}\right) \\
+ & \left(\frac{\sigma \xi_{2}}{\left(1+\xi_{1}\right)}-\frac{\sigma^{*} \xi_{1}}{\left(1+\xi_{2}\right)}\right)\left(\frac{2 n_{1} \sigma}{\left(1+\xi_{2}\right)^{2}\left(1+\xi_{1}\right)}+\frac{2 n_{2} \sigma^{*}}{\left(1+\xi_{1}\right)^{2}\left(1+\xi_{2}\right)}\right) .
\end{aligned}
$$


where $\xi_{1}=\sigma(\lambda-\log x)$ and $\xi_{2}=\sigma^{*}\left(\lambda^{*}-\log x\right)$. Now, as $\sigma \geq(\leq) \sigma^{*}$ and $\lambda \geq(\leq) \lambda^{*}$, implying that $\sigma(\lambda-\log x) \geq(\leq) \sigma^{*}\left(\lambda^{*}-\log x\right)$ i.e. $\xi_{1} \geq(\leq) \xi_{2}$, and moreover, $\frac{\xi}{1+\xi}=1-\frac{1}{1+\xi}$ is increasing in $\xi$, then $\frac{\xi_{2}^{2}}{\left(1+\xi_{2}\right)^{2}} \leq(\geq) \frac{\xi_{1}^{2}}{\left(1+\xi_{1}\right)^{2}}$. Again,

$$
\begin{aligned}
\frac{\sigma \xi_{2}}{1+\xi_{1}}-\frac{\sigma^{*} \xi_{1}}{1+\xi_{2}} & =\frac{\sigma \sigma^{*}\left\{\left(\lambda^{*}-\log x\right)\left(1+\sigma^{*}\left(\lambda^{*}-\log x\right)\right)-(\lambda-\log x)(1+\sigma(\lambda-\log x))\right\}}{\left(1+\xi_{1}\right)\left(1+\xi_{2}\right)} \\
\leq(\geq) & 0 .
\end{aligned}
$$

So, by Lemma 3.1 (Lemma 3.3) of Kundu et al. ([9]), the result is proved.

Theorem 3.3 guarantees that, for two $n$ component parallel systems (with a sufficient condition) having independent LL distributed lifetimes with a common scale parameter vector, the majorized shape parameter vector leads to greater system's lifetime in the sense of likelihood ratio order. The next theorem states that the majorized scale parameter vector leads to smaller system's lifetime in the sense of likelihood ratio order when the shape parameter vector of these two $n$-component parallel systems are common.

Theorem 3.5 For $i=1,2, \ldots, n$, let $X_{i}$ and $Y_{i}$ be two sets of mutually independent random variables with $X_{i} \sim L L\left(\sigma_{i}, \lambda_{i}\right)$ and $Y_{i} \sim L L\left(\sigma_{i}, \delta_{i}\right)$. Further, suppose that $\boldsymbol{\sigma} \in \mathcal{E}_{+}, \boldsymbol{\lambda}, \boldsymbol{\delta} \in \mathcal{D}_{+}$ or $\boldsymbol{\sigma} \in \mathcal{D}_{+}, \boldsymbol{\lambda}, \boldsymbol{\delta} \in \mathcal{E}_{+}$. Then,

$$
\boldsymbol{\lambda} \stackrel{m}{\succeq} \boldsymbol{\delta} \text { implies } X_{n: n} \leq_{l r} Y_{n: n}
$$

Proof: In view of Theorem 3.2 and using (3.1) and (3.2), we are to prove that

$$
\eta_{1}(x)=\frac{\sum_{k=1}^{n} \frac{\sigma_{k}}{x}\left(1-\frac{1}{1+\sigma_{k}\left(\lambda_{k}-\log x\right)}\right)}{\sum_{k=1}^{n} \frac{\sigma_{k}}{x}\left(1-\frac{1}{1+\sigma_{k}\left(\delta_{k}-\log x\right)}\right)}
$$

is decreasing in $x$ i.e. to prove that

$$
\psi_{2}(\boldsymbol{\lambda}, x)=\frac{\sum_{k=1}^{n} \frac{\sigma_{k}^{2}}{\left(1+\sigma_{k}\left(\lambda_{k}-\log x\right)\right)^{2}}}{\sum_{k=1}^{n} \frac{\sigma_{k}^{2}\left(\lambda_{k}-\log x\right)}{\left(1+\sigma_{k}\left(\lambda_{k}-\log x\right)\right)}}
$$

is Schur-convex in $\boldsymbol{\lambda}$. Now,

$$
\frac{\partial \psi_{2}}{\partial \lambda_{i}} \stackrel{\text { sign }}{=}-\frac{2 \sigma_{i}^{3}}{\left(1+\sigma_{i}\left(\lambda_{i}-\log x\right)\right)^{3}} \sum_{k=1}^{n} \frac{\sigma_{k}^{2}\left(\lambda_{k}-\log x\right)}{\left(1+\sigma_{k}\left(\lambda_{k}-\log x\right)\right)}-\frac{\sigma_{i}^{2}}{\left(1+\sigma_{i}\left(\lambda_{i}-\log x\right)\right)^{2}} \sum_{k=1}^{n} \frac{\sigma_{k}^{2}}{\left(1+\sigma_{k}\left(\lambda_{k}-\log x\right)\right)^{2}} .
$$

So, by noticing the fact that

$$
\frac{\partial}{\partial \sigma_{i}}\left[\frac{\sigma_{i}}{\left(1+\sigma_{i}\left(\lambda_{i}-\log x\right)\right)}\right]=\frac{1}{\left(1+\sigma_{i}\left(\lambda_{i}-\log x\right)\right)^{2}}>0
$$

giving that $\frac{\sigma_{i}}{\left(1+\sigma_{i}\left(\lambda_{i}-\log x\right)\right)}$ is increasing in $\sigma_{i}, \boldsymbol{\lambda} \in \mathcal{D}_{+}\left(\right.$resp. $\left.\mathcal{E}_{+}\right)$and $\boldsymbol{\sigma} \in \mathcal{E}_{+}\left(\right.$resp. $\left.\mathcal{D}_{+}\right)$, i.e. for all $i \leq j \lambda_{i} \geq(\leq) \lambda_{j}$ and $\sigma_{i} \leq(\geq) \sigma_{j}$ gives

$$
\frac{\sigma_{i}^{3}}{\left(1+\sigma_{i}\left(\lambda_{i}-\log x\right)\right)^{3}} \leq(\geq) \frac{\sigma_{j}^{3}}{\left(1+\sigma_{j}\left(\lambda_{i}-\log x\right)\right)^{3}} \leq(\geq) \frac{\sigma_{j}^{3}}{\left(1+\sigma_{j}\left(\lambda_{j}-\log x\right)\right)^{3}}
$$


and

$$
\frac{\sigma_{i}^{2}}{\left(1+\sigma_{i}\left(\lambda_{i}-\log x\right)\right)^{2}} \leq(\geq) \frac{\sigma_{j}^{2}}{\left(1+\sigma_{j}\left(\lambda_{i}-\log x\right)\right)^{2}} \leq(\geq) \frac{\sigma_{j}^{2}}{\left(1+\sigma_{j}\left(\lambda_{j}-\log x\right)\right)^{2}}
$$

So,

$$
\frac{\partial \psi_{2}}{\partial \lambda_{i}}-\frac{\partial \psi_{2}}{\partial \lambda_{j}} \geq(\leq) 0
$$

Thus the result follows from Lemma 3.1 (Lemma 3.3) of Kundu et al. ([9]).

\section{References}

[1] Boland, P. J. and El-Neweihi, E. (1998). Statistical and information based (physical) minimal repair for k-out-of-n systems. Journal of Applied Probability, 35, 731-740.

[2] Dykstra, R., Kochar, S.C., and Rojo, J. (1997). Stochastic comparisons of parallel systems of heterogeneous exponential components. Journal of Statistical Planning and Inference, 65, 203-211.

[3] El-Neweihi, E., Proschan, F. and Sethuraman, J. (1986). Optimal Allocation of Components in Parallel-Series and Series-Parallel Systems. Journal of Applied Probability, 23, 770-777.

[4] Fang, L. and Zhang, X. (2015). Stochastic comparisons of parallel systems with exponentiated Weibull components. Statistics and Probability Letters, 97, 25-31.

[5] Ghitany, M.E. (2004). The monotonicity of the reliability measures of the beta distribution. Appl. Math. Lett., 17 (11), 1277-1283.

[6] Gómez-Déniz, E, Sordo, M.A. and Calderín-Ojeda, E (2014). The Log-Lindley distribution as an alternative to the beta regression model with applications in insurance. Insurance: Mathematics and Economics, 54, 49-57.

[7] Gupta, N., Patra, L.K. and Kumar, S. (2015). Stochastic comparisons in systems with Fréchet distributed components. Operations Research Letters, 43(6), 612-615.

[8] Kundu, A. and Chowdhury, S. (2016): Ordering properties of order statistics from heterogeneous exponentiated Weibull models. Statistics and Probability Letters, 114, 119-127.

[9] Kundu, A., Chowdhury, S., Nanda, A. and Hazra, N. (2016): Some Results on Majorization and Their Applications. Journal of Computational and Applied Mathematics, 301, 161-177.

[10] Lai, C.D. and Jones, G. (2015). Beta hazard rate distribution and applications. IEEE Transactions on Reliability, 64, 44-50. 
[11] Lai, C.D., and Mukherjee, S.P. (1986). A note on 'A finite range distribution of failure times'. Microelectronics Reliability, 26(1), 183-189.

[12] Lariviere, M.A. (2006). A note on probability distributions with increasing generalized failure rates. Operations Research, 54, 602-604.

[13] Lariviere, M.A. and Porteus, E.L. (2001). Selling to a newsvendor: an analysis of price-only contracts. Manufacturing \& Service Operations Management, 3, 292-305.

[14] Lindley, D.V. (1958). Fiducial distributions and Bayes theorem. Journal of the Royal Statistical Society: Series B, 20(1), 102-107.

[15] Marshall, A.W., Olkin, I., and Arnold, B.C. (2011). Inequalities: Theory of Majorization and Its Applications. Springer series in Statistics, New York.

[16] Misra, N. and Misra, A.K. (2013). On comparison of reversed hazard rates of two parallel systems comprising of independent gamma components. Statistics and Probability Letters, 83, $1567-1570$.

[17] Moore, T., and Lai, C.D. (1994). The beta failure rate distribution, Proc. 30th Annu. Conf. Oper. Res. Soc. NZ/45th Annu. Conf. New Zealand Stat. Assoc., Palmerston North, New Zealand, 339-344.

[18] Mukherjee, S.P. and Islam, A (1986). A finite-range distribution of failure times Naval Research Logistics, 30 (3), 487-491.

[19] Shaked, M. and Shanthikumar, J.G. (2007). Stochastic Orders. Springer, New York.

[20] Torrado, N. and Kochar, S.C. (2015). Stochastic order relations among parallel systems from Weibull distributions. Journal of Applied Probability, 52, 102-116.

[21] Zakerzadeh, H. and Dolati, A. (2010). Generalized Lindley distribution. Journal of Mathematical Extension, 3(2), 13-25.

[22] Ziya, S., Ayhan, H., and Foley, R.D. (2004). Relationships among three assumptions in revenue management. Operations Research, 52, 804-809.

[23] Zhao, P. and Balakrishnan, N. (2011). New results on comparison of parallel systems with heterogeneous gamma components. Statistics and Probability Letters, 81, 36-44. 